\title{
Togitare
Enfermagem
}

\section{PARTICIPAÇÃO DO COMPANHEIRO DA GESTANTE NAS CONSULTAS DE PRÉ-NATAL: PREVALÊNCIA E FATORES ASSOCIADOS}

\author{
Jaqueline Guimarães Elói de Brito ${ }^{1}$ \\ José Marcos de Jesus Santos ${ }^{2}$ (i) \\ Maria do Socorro Claudino Barreiro ${ }^{1}$ \\ Diego da Silva Dantas ${ }^{1}$ \\ Adriana Moraes Leite ${ }^{2}$ \\ Rosemar Barbosa Mendes ${ }^{1}$ (i)
}

\begin{abstract}
RESUMO
Objetivo: identificar a prevalência e fatores associados à participação do companheiro da gestante no pré-natal.

Método: estudo transversal realizado entre março e julho de 2018 por meio de entrevista com 655 puérperas de uma regional do Nordeste brasileiro. Estimou-se associações com uso do Quiquadrado e Razão de Prevalência.

Resultados: dentre mulheres com companheiro e que realizaram pré-natal $(85,6 \% ; n=561)$, a participação do parceiro foi de $(44,2 \% ; n=248)$, sendo maior entre aquelas que planejaram a gravidez (RP: 1,25; IC 95\%: 1,07-2,10), desejaram engravidar (RP: 1,22; IC 95\%: 1,01-1,98), iniciaram precocemente $o$ acompanhamento (RP: 1,31 ; IC $95 \%: 1,01-2,46$ ) e realizaram seis ou mais consultas (RP: 1,49; IC 95\%: 1,32-1,81). Houve menor participação entre mulheres com baixa escolaridade (RP: 0,72; IC 95\%: 0,39-0,77) e que utilizaram serviço público (RP: 0,65; IC 95\%: 0,24-0,85).

Conclusão: a baixa prevalência de participação do companheiro da gestante no pré-natal evidencia a necessidade de maior estímulo à sua inclusão neste processo.
\end{abstract}

DESCRITORES: Gravidez; Cuidado Pré-Natal; Paternidade; Saúde do Homem; Saúde Pública.

\section{PARTICIPACIÓN DEL COMPAÑERO DE LA EMBARAZADA EN LAS CONSULTAS PRENATALES: PREVALENCIA Y FACTORES ASOCIADOS}

\section{RESUMEN:}

Objetivo: identificar la prevalencia y los factores asociados a la participación del acompañante de la gestante en el prenatal. Método: estudio transversal realizado entre marzo y julio de 2018 mediante una entrevista con 655 puérperas de un hospital regional del Nordeste de Brasil. Las asociaciones se estimaron mediante la Chi-cuadrado y la Razón de Prevalencia. Resultados: entre las mujeres con pareja que tuvieron control prenatal $(85,6 \% ; n=561)$, la participación de la pareja fue (44,2\%; $n=248)$, siendo mayor entre las que planificaron el embarazo (PR: 1,25; IC $95 \%$ : 1,07-2,10), deseaban quedarse embarazadas (PR: 1,22; IC 95\%: 1,01-1,98), iniciaron precozmente un seguimiento (PR: 1,31; IC 95\%: 1,01-2,46) y tenían seis o más consultas (PR: 1,49; IC 95\%: 1,32-1,81). La participación fue menor entre las mujeres con bajo nivel educativo (RP: 0,72; IC 95\%: 0,39-0,77) y que utilizaron los servicios públicos (RP: 0,65; IC 95\%: 0,24-0,85). Conclusión: la baja prevalencia de participación del acompañante de la gestante en el prenatal evidencia la necesidad de un mayor estímulo a su inclusión en este proceso.

DESCRIPTORES: Embarazo; Atención Prenatal; Paternidad; Salud del Hombre; Salud Pública. 
A assistência pré-natal visa o desenvolvimento de uma gestação saudável a partir da abordagem de aspectos biopsicossociais, diagnósticos e terapêuticos e oferta de atividades educativas e preventivas cabíveis neste processo ${ }^{(1-2)}$. Historicamente, as ações em saúde voltadas ao período gravídico-puerperal foram direcionadas exclusivamente à mulher e ao feto/recém-nascido, com notável diferença no foco da promoção da saúde ao binômio mãe-bebê e à saúde masculina. Há também um entrave cultural motivado por estereótipos na visitação de homens aos serviços de saúde, em especial da Atenção Primária ${ }^{(3-4)}$.

No Brasil, o Ministério da Saúde publicou em 2016 um Guia do Pré-Natal do Parceiro para Profissionais da Saúde, que estabelece: $1^{\circ}$ incentivo à participação do homem nas consultas de pré-natal e nas atividades educativas; $2^{\circ}$ realização de testes rápidos e exames de rotina no parceiro (tipagem sanguínea e fator $\mathrm{RH}, \mathrm{HBsAg}$, teste treponêmico e/ ou não treponêmico para detecção de sífilis, pesquisa de anticorpos anti-HIV e anti-HCV, hemograma, lipidograma, dosagem de glicose, eletroforese da hemoglobina, aferição de pressão arterial, verificação de peso e cálculo de $\mathrm{IMC}$ ); $3^{\circ}$ atualização do cartão de vacina do parceiro; $4^{\circ}$ abordagens de temáticas voltados ao público masculino; e $5^{\circ}$ orientações sobre o papel do homem na gestação, pré-parto, parto, puerpério imediato e cuidados com a criança $a^{(3)}$.

Todavia, embora a participação do parceiro da gestante nas consultas de pré-natal tenha passado a ser estimulada nos últimos anos no país, muitos homens continuam sem entender a importância e/ou finalidade de participarem deste processo. Autores atribuem essa problemática aos profissionais de saúde da Atenção Primária, quando ignoram e/ou desqualificam a participação do parceiro na gestação. Muitos parceiros nem chegam a ser convidados para entrar na sala onde são realizados os atendimentos à mulher $r^{(5-6)}$.

Cabe destacar que a participação do homem no pré-natal é fator determinante para a criação e fortalecimento de vínculos afetivos saudáveis, além de favorecer a realização de um pré-natal com melhores indicadores de qualidade ${ }^{(3)}$. A presença do parceiro no pré-natal pode contribuir para um maior número de consultas realizadas ${ }^{(2)}$, bem como influenciar na saúde física e psicológica da mulher e criança, reduzir a ansiedade, aliviar a dor, diminuir a duração do trabalho de parto, favorecer o aleitamento materno e reduzir os índices de violências domésticas e/ou obstétricas ${ }^{(6-8)}$.

Acrescenta-se, por fim, que a Política Nacional de Atenção Integral à Saúde do Homem (PNAISH) também defende o envolvimento dos pais no ciclo gravídico-puerperal, buscando consolidar a mudança crucial do foco no binômio mãe-criança para o trinômio pai-mãe-criança ${ }^{(3,9-10)}$. Frente ao exposto, o objetivo deste estudo foi identificar a prevalência e os fatores associados à participação do companheiro das gestantes nas consultas de prénatal.

\section{MÉTODO}

Trata-se de um estudo transversal, descritivo e inferencial, realizado entre março e julho de 2018. Foram avaliadas 655 puérperas por meio de entrevista e visualizaçăo do cartão de pré-natal durante o pós-parto imediato. O local de realização foi uma maternidade de risco habitual localizada em Lagarto, Sergipe, Brasil. A instituição possui quatro leitos obstétricos particulares, seis leitos de pré-parto e 31 leitos de alojamento conjunto. Atende partos com financiamento público e/ou privado de parturientes de risco habitual provenientes de Lagarto e de outras cidades da Região Centro-Sul do estado. 
A população elegível ao estudo era composta por 1.250 mulheres com base na estimativa anual de partos disponibilizada pela direção da instituição. A partir disso foi feito cálculo amostral usando a fórmula de Barbetta (2014), sendo considerado um nível de confiança de $97 \%$ e de erro amostral de $3 \%{ }^{(11)}$. Acrescentou-se uma margem de segurança de $10 \%$ no número calculado, resultando em 655 puérperas.

As participantes foram selecionadas por amostragem aleatória simples, a partir de uma listagem de internação diária, sendo consideradas elegíveis todas as mulheres que tiveram parto de feto vivo de qualquer peso ou idade gestacional. Não foram incluídas mulheres que não falassem e/ou compreendessem o idioma português e/ou que apresentassem transtornos mentais graves.

Para a coleta dos dados, foram aplicados questionários durante entrevistas com as puérperas, respeitando-se um intervalo mínimo de seis horas após o parto. O questionário abordava questões sociodemográficas maternas, relacionadas à gravidez e ao pré-natal da mulher e do parceiro.

Destaca-se que foram estimadas associações entre variáveis sociodemográficas maternas (faixa etária, raça, zona de moradia, escolaridade e trabalho remunerado), relacionadas à gravidez (planejamento reprodutivo, sentimento quanto à gravidez e percepção do tempo da gestação) e ao pré-natal (trimestre de início, número de consultas, acompanhamento pelo mesmo profissional e tipo de serviço) com a participação do parceiro das gestantes nestas consultas.

A análise estatística foi realizada no IBM® SPSS 20.0 Mac. Utilizou-se as técnicas uni e bivariada para obtenção da distribuição dos valores das frequências absoluta e relativa/ proporcional. As associações foram estimadas por meio do teste Qui-quadrado entre variáveis categóricas, com uso da Razão de Prevalência como medida de associação e seus respectivos intervalos de confiança de $95 \%$ (IC 95\%). Em todos os casos foi adotada significância de $5 \%$.

Este estudo está vinculado ao Projeto Nascer em Lagarto, SE: Inquérito Municipal sobre Parto e Nascimento, aprovado pelo Comitê de Ética em Pesquisa da Universidade Federal de Sergipe, sob parecer $n^{\circ} 2.553 .774$. Os pesquisadores seguiram as diretrizes e normas regulamentadoras preconizadas na Resolução $N^{\circ} 466 / 12$ do Conselho Nacional de Saúde sobre as pesquisas envolvendo seres humanos ${ }^{(12)}$.

\section{RESULTADOS}

Dentre as mulheres com companheiro à época da pesquisa $(85,6 \% ; n=561),(99,8 \%$; $n=560)$ referiram a realização do pré-natal. Entretanto, somente $(44,2 \% ; n=248)$ delas tiveram a participação do parceiro nestas consultas, sendo $(33,1 \% ; n=82)$ com participação total/em todas as consultas e $(66,9 \% ; n=166)$ com participação parcial/algumas consultas.

A análise de associações entre as características sociodemográficas maternas e a participação do parceiro no pré-natal mostrou uma menor prevalência entre mulheres com baixa escolaridade (RP: 0,72; IC 95\%: 0,39-0,77) (Tabela 1). 
Tabela 1 - Associações entre variáveis sociodemográficas maternas e a participação do parceiro no prénatal $(n=560)$. Região Centro-Sul de Sergipe, Brasil, 2018

\begin{tabular}{|c|c|c|c|c|}
\hline \multirow[t]{2}{*}{ Variáveis sociodemográficas } & \multicolumn{2}{|c|}{ Participação do parceiro no pré-natal } & \multirow[t]{2}{*}{ Valor de $\mathbf{p}$} & \multirow{2}{*}{$\begin{array}{c}\text { RP } \\
\text { (IC 95\%) }\end{array}$} \\
\hline & $\operatorname{Sim}(\%)(n=248)$ & Não (\%) (n=312) & & \\
\hline \multicolumn{5}{|l|}{ Faixa etária } \\
\hline$\leq 19$ anos & 45,5 & 54,5 & \multirow{2}{*}{0,861} & 1,02 \\
\hline$\geq 20$ anos & 44,4 & 55,6 & & $(0,65-1,64)$ \\
\hline \multicolumn{5}{|l|}{ Raça/Cor da pele } \\
\hline Branca/Amarela & 50 & 50 & \multirow{2}{*}{0,267} & 1,15 \\
\hline Preta/Parda & 43,6 & 56,4 & & $(0,82-2,04)$ \\
\hline \multicolumn{5}{|l|}{ Zona de moradia } \\
\hline Rural & 41,6 & 58,4 & \multirow{2}{*}{0,169} & 0,88 \\
\hline Urbana & 47,4 & 52,6 & & $(0,80-1,77)$ \\
\hline \multicolumn{5}{|l|}{ Escolaridade } \\
\hline Analfabeta/Fundamental & 36,7 & 63,3 & \multirow{2}{*}{0,001} & 0,72 \\
\hline Médio/Superior & 51,1 & 48,9 & & $(0,39-0,77)$ \\
\hline \multicolumn{5}{|l|}{ Possui trabalho remunerado } \\
\hline Sim & 49,1 & 50,9 & \multirow{2}{*}{0,176} & 1,15 \\
\hline Não & 42,8 & 57,2 & & $(0,89-1,86)$ \\
\hline
\end{tabular}

Legenda: RP= Razão de Prevalência, IC 95\%= Intervalo de Confiança de 95\%.

A gravidez planejada (RP: 1,25; IC 95\%: 1,07-2,10) e o desejo materno de realmente engravidar naquele período da vida (RP: 1,22; IC 95\%: 1,01-1,98) mostraram-se associadas à participação do parceiro no pré-natal (Tabela 2).

Tabela 2 - Associações entre variáveis relacionadas à gravidez e a participação do parceiro na assistência pré-natal ( $n=560)$. Região Centro-Sul de Sergipe, Brasil, 2018 (continua)

\section{Variáveis relacionadas à gravidez}

Participação do parceiro no pré-natal Valor de p
\[ \operatorname{Sim}(\%)(n=248) \quad \text { Não (\%) }(n=312) \]

0,017

60,3

$39,7 \quad 60,3$

Sim

Não

Sentimento em relação à gravidez

\section{Satisfeita}

Mais ou menos/Insatisfeita

46

41,1

54

58,9
RP

(IC 95\%)

Percepção de tempo da gestação 


\begin{tabular}{lllll}
\hline Queria engravidar agora & 49,1 & 50,9 & $\mathbf{0 , 0 4 1}$ & 1,22 \\
\cline { 1 - 2 } Queria engravidar depois & 40,4 & 59,6 & & $(1,01-1,98)$
\end{tabular}

Legenda: RP= Razão de Prevalência, IC 95\%=Intervalo de Confiança de 95\%.

Também foi observada uma maior prevalência de participação do parceiro da gestante nas consultas de pré-natal entre mulheres que iniciaram precocemente seu acompanhamento (RP: 1,31 ; IC $95 \%: 1,01-2,46$ ) e que realizaram seis ou mais consultas (RP: 1,$49 ;$ IC 95\%: 1,32-1,81), e menor entre aquelas que utilizaram o serviço público neste processo (RP: 0,65; IC 95\%: 0,24-0,85) (Tabela 3).

Tabela 3 - Associações entre as características da assistência pré-natal e a participação do parceiro nestas consultas ( $n=560)$. Região Centro-Sul de Sergipe, Brasil, 2018

\begin{tabular}{|c|c|c|c|c|}
\hline \multirow{2}{*}{$\begin{array}{l}\text { Variáveis da assistência pré- } \\
\text { natal }\end{array}$} & \multicolumn{2}{|c|}{ Participação do parceiro no pré-natal } & \multirow{2}{*}{$\begin{array}{l}\text { Valor de } \\
\text { p }\end{array}$} & \multirow{2}{*}{$\begin{array}{c}\text { RP } \\
\text { (IC 95\%) }\end{array}$} \\
\hline & $\operatorname{Sim}(\%)(n=248)$ & Não (\%) (n=312) & & \\
\hline \multicolumn{5}{|l|}{ Início precoce } \\
\hline Sim & 46,6 & 53,4 & \multirow[t]{2}{*}{0,046} & 1,31 \\
\hline Não & 35,6 & 64,4 & & $(1,01-2,46)$ \\
\hline \multicolumn{5}{|l|}{ Número de consultas } \\
\hline 6 ou mais consultas & 47,7 & 52,3 & \multirow[t]{2}{*}{0,004} & 1,49 \\
\hline$\leq 5$ consultas & 32 & 68 & & $(1,32-1,81)$ \\
\hline \multicolumn{5}{|c|}{ Acompanhamento pelo mesmo profissional } \\
\hline Sim & 45,6 & 54,4 & \multirow[t]{2}{*}{0,502} & 1,07 \\
\hline Não & 42,6 & 57,4 & & $(0,79-1,59)$ \\
\hline
\end{tabular}

Tipo de serviço que realizou a maioria das consultas

\begin{tabular}{lllll}
\hline Público & 41,5 & 58,5 & $<0,001$ & 0,65 \\
\cline { 1 - 2 } Privado & 64,1 & 35,9 & $(0,24-0,85)$
\end{tabular}

Legenda: RP= Razão de Prevalência, IC 95\%= Intervalo de Confiança de 95\%.

\section{DISCUSSÃO}

Os benefícios da assistência pré-natal têm ampla discussão no meio científico, embora abordados em perspectivas exclusivamente voltadas à mãe-bebê $\hat{e}^{(13)}$. Desta maneira, em um dos eixos da PNAISH foi desenvolvida a Estratégia Pré-Natal do Parceiro, que objetiva engajar o envolvimento dos homens no acompanhamento pré-natal, parto e pós-parto(9). Esse cenário justifica a produção de evidências científicas que contribuam para encorajar a prática de atenção ao pré-natal do parceiro, tendo em vista o empoderamento familiar ${ }^{(3)}$. 
No presente estudo, foi evidenciado que $44,2 \%$ das mulheres entrevistadas tiveram a participação do companheiro nas consultas de pré-natal, com somente $14,6 \%$ participando de fato em todas as consultas. Esse resultado se diferencia do achado de uma pesquisa de nível nacional feita pelo Ministério da Saúde entre 2017/2018 com 37.322 homens, na qual $72,2 \%$ dos participantes referiram ter acompanhado suas companheiras nas consultas de pré-natal ${ }^{(14)}$. Ademais, em contexto internacional, um estudo realizado com 5.333 mulheres na Inglaterra mostrou que mais de $80 \%$ dos parceiros acompanharam a gestação, parto e puerpério de suas esposas ${ }^{(8)}$.

No Sergipe, um dos principais motivos relatados pelos pais para não comparecerem às Unidades Básicas de Saúde (UBS) foi a necessidade de trabalhar (76\%)(15), resultado semelhante ao encontrado em outros estudos ${ }^{(16-17)}$. Ressalta-se que a Lei $n^{\circ} 13.257 / 2016$ dá o direito ao trabalhador de se ausentar do serviço por até dois dias para acompanhar consultas e exames complementares, sem prejuízo salarial(18).

Todavia, mesmo amparados por lei, muitos trabalhadores têm receio de faltar no serviço por razões de saúde, fato que, somado à pouca resolutividade dos serviços e/ou ao longo tempo de espera para atendimentos, dificultam a ida dos homens aos serviços de saúde da atenção básica e/ou especializada do país ${ }^{(6)}$.

Ademais, quando os companheiros se fazem presentes, em muitos casos, não são realizadas atividades que os incluam, fazendo com que o homem não se sinta convidado para o pré-natal(5-6). É evidente que a maioria dos pré-natalistas ainda estão focando suas orientações exclusivamente na gestante $(69,1 \%)$, o que mostra também uma invisibilidade do pai, mesmo quando este se faz presente no serviço de saúde ${ }^{(5-6,15)}$.

Reforça-se que o Ministério da Saúde preconiza a oferta de algumas atividades preventivas e diagnósticas para os companheiros das gestantes durante o pré-natal. Dentre elas, cita-se a solicitação de testes rápidos, HIV, Sífilis e Hepatites; aconselhamentos e exames de rotina, e atualização do cartão de vacina ${ }^{(3)}$, além da participação em atividades educativas para estimular os pais a participarem ativamente do cuidado com o filho ${ }^{(19)}$.

Foi também identificado um perfil característico de mulheres e da assistência prénatal que melhor favorecem o envolvimento do parceiro no acompanhamento gestacional: mulheres que desejaram ou planejaram a gravidez e/ou que iniciaram precocemente 0 acompanhamento pré-natal e/ou que realizaram seis ou mais consultas e/ou mulheres com maior escolaridade e/ou que utilizaram o serviço privado.

Autores apresentam a escolaridade como fator importante na utilização dos serviços de saúde pelas camadas da população menos favorecidas ${ }^{(2)}$. Quando o nível de escolaridade do casal é mais elevado, há uma maior adesão por parte da mulher ao pré-natal, e a participação do homem durante o ciclo gravídico comumente é mais ativa ${ }^{(17)}$.

Além disso, outro estudo encontrou que o envolvimento paterno é maior entre mulheres autodeclaradas brancas/amarelas ${ }^{(8)}$. Isso pode ser explicado, inclusive, por um possível racismo institucional na saúde, uma vez que mulheres pardas/negras têm menor acesso aos serviços de qualidade, à atenção ginecológica e obstétrica(20).

A limitação deste estudo está relacionada à obtenção dos dados exclusivamente a partir do relato das puérperas entrevistadas, sem a obtenção dos dados diretamente com os parceiros das gestantes nas consultas de pré-natal. 
nas consultas de pré-natal, com associação de variáveis sociodemográficas maternas e características assistenciais. Os fatores associados à maior participação do companheiro das gestantes no pré-natal foram: alta escolaridade materna, utilização do serviço privado, gravidez planejada, desejo materno de realmente engravidar, início precoce do pré-natal e realização de seis ou mais consultas.

Esses achados reforçam a necessidade de maior estímulo à inclusão do homem neste processo assistencial. Recomenda-se, em especial aos profissionais da Enfermagem, atenção ao fato de a gravidez também ser um assunto de homem, de modo que estimular a participação do pai/parceiro durante todo esse processo pode ser fundamental para o bem-estar biopsicossocial da mãe, do bebê e dele próprio.

Reforça-se a necessidade de sensibilizar e preparar as equipes de saúde para receberem e acolherem os homens de forma integral e adequada a este seguimento. $O$ enfermeiro ocupa um papel fundamental neste aspecto, pois deve orientar e estimular as gestantes quanto à possibilidade e importância da presença do parceiro no prénatal, não oferecendo obstáculos à sua participação, e sim uma escuta qualificada sobre as expectativas do casal em relação à paternidade/maternidade. Desse modo, o pai se sentirá seguro para oferecer o apoio necessário à mulher e à criança, visto que entenderá as alterações fisiológicas/emocionais pertinentes ao ciclo gravídico-puerperal no qual está inserido.

\section{REFERÊNCIAS}

1. Gonçalves ITJP, Souza KV, Amaral MA, Oliveira ARS de, Ferreira WFC. Prática do Acolhimento na Assistência Pré-Natal: limites, potencialidades e contribuições da enfermagem. Rev Rene [Internet]. 2013 [acesso em 24 mar 2019]; 14(3). Disponível em: http://www.periodicos.ufc.br/rene/article/view/3503/2744.

2. Rosa CQ da, Silveira DS da, Costa JSD da. Factors associated with lack of prenatal care in a large municipality. Rev. Saúde Pública [Internet]. 2014 [acesso em 13 abr 2019]; 48(6). Disponível em: http:// dx.doi.org/10.1590/S0034-8910.2014048005283.

3. Ministério da Saúde (BR). Secretaria de Atenção à Saúde. Departamento de Ações Programáticas Estratégicas. Guia do Pré-Natal do Parceiro para Profissionais de Saúde. [Internet]. Brasília: Ministério da Saúde; 2016. [acesso em 13 abr 2019]. Disponível em: http://portal.saude.pe.gov.br/sites/portal.saude. pe.gov.br/files/guia prenataldoparceiro 1.pdf.

4. Moreira MCN, Gomes R, Ribeiro CR. E agora o homem vem?! Estratégias de atenção à saúde dos homens. Cad. Saúde Pública [Internet]. 2016 [acesso em 21 fev 2019]; 32(4). Disponível em: http://dx.doi. org/10.1590/0102-311X00060015.

5. Ribeiro CR, Gomes R, Moreira MCN. Fatherhood and parenting as health issues facing the rearrangements of gender. Ciênc. saúde coletiva [Internet]. 2015 [acesso em 12 jan 2019]; 20(11). Disponível em: http://dx.doi.org/10.1590/1413-812320152011.19252014.

6. Ribeiro CR, Gomes R, Moreira MCN. Encontros e desencontros entre a saúde do homem, a promoção da paternidade participativa e a saúde sexual e reprodutiva na atenção básica. Physis [Internet]. 2017 [acesso em 09 abr 2019]; 27(1). Disponível em: http://dx.doi.org/10.1590/s0103-73312017000100003.

7. Branco VMC. Profissionais de saúde: parceiros na construção de uma sociedade mais solidária. In: Unidade de Saúde Parceira do Pai. [Internet]. Rio de Janeiro: Secretaria Municipal de Saúde; 2009. [acesso em 09 abr 2019]. Disponível em: http://primeirainfancia.org.br/wp-content/uploads/2016/04/ unidade-de-sac3bade-parceira-do-pai.pdf.

8. Redshaw M, Henderson J. Fathers' engagement in pregnancy and childbirth: evidence from a national survey. BMC Pregnancy and Childbirth [Internet]. 2013 [acesso em 02 mar 2019]; 13(70). Disponível em: 
9. Chakora ES. National Policy for Full Attention to Men's Health. Esc Anna Nery [Internet]. 2014 [acesso em 02 mar 2019]; 18(4). Disponível em: http://doi.org/10.5935/1414-8145.20140079.

10. Teixeira RC, Mandú ENT, Corrêa AC de P, Marcon SS. Vivências e necessidades de saúde de homens no períoso pós-nascimento de um filho. Rev. bras. enferm. [Internet]. 2014 [acesso em 02 fev 2019]; 67(5). Disponível em: http://dx.doi.org/10.1590/0034-7167.2014670516.

11. Barbetta PA. Estatística aplicada às ciências sociais. 9. ed. Santa Catarina: UFSC; 2014.

12. Ministério da Saúde (BR). Conselho Nacional de Saúde. Diretrizes e normas regulamentadoras de pesquisa envolvendo seres humanos. Resolução n. 466, de 12 de dezembro de 2012. Brasília; 2012.

13. Ferreira IS, Fernandes AFC, Lô KKR, Melo TP de, Gomes AMF, Andrade IS. Perceptions of pregnant women about the role of partners in prenatal consultations. Rev Rene [Internet]. 2016 [acesso em 28 jan 2019]; 17(3). Disponível em: http://dx.doi.org/10.15253/2175-6783.2016000300003.

14. Ministério da Saúde (BR). Secretaria de Atenção à Saúde. Departamento de Ações Programáticas Estratégicas. Relatório da Pesquisa Saúde do Homem, Paternidade e Cuidado Brasil - III etapa. [Internet] Brasília: Ministério da Saúde; 2018. [acesso em 09 abr 2019]. Disponível em: http://portalarquivos2.saude. gov.br/images/pdf/2018/agosto/16/Relatorio-Brasil-Pesquisa-Saude-do-Homem-Ill-etapa.pdf.

15. Ministério da Saúde (BR). Secretaria de Atenção à Saúde. Departamento de Ações Programáticas Estratégicas. II Relatório da Pesquisa Saúde do Homem, Paternidade e Cuidado Sergipe. [Internet] Brasília: Ministério da Saúde; 2017 [acesso em 09 abr 2019]. Disponível em: http://portalarquivos2.saude. gov.br/images/pdf/2018/agosto/22/ETAPA-II/SE.pdf.

16. Cardoso VEPS, Silva Junior AJ da, Bonatti AF, Santos GWS dos, Ribeiro TAN. The partner's involvement in the prenatal routine through the pregnant women perspective. Rev Fund Care Online [Internet]. 2018 [acesso em 28 jan 2019]; 10(3). Disponível em: http://dx.doi.org/10.9789/2175-5361.2018. v10i3.856-862.

17. Caldeira LA, Ayres LFA, Oliveira LVA, Henriques BD. The point of view of pregnant women about the participation of man in the gestational process. Rev Enferm Centr-Oeste Min [Internet]. 2017 [acesso em 28 jan 2019]; 7. Disponível em: http://dx.doi.org/10.19175/recom.v7i0.1417.

18. Gomes R, Albernaz L, Ribeiro CRS, Moreira MCN, Nascimento M. Lines of male care geared to sexual health, reproduction and paternity. Ciênc. saúde coletiva [Internet]. 2016 [acesso em 28 jan 2019]; 21(5). Disponível em: http://dx.doi.org/10.1590/1413-81232015215.26842015.

19. Yeganeh N, Simon M, Mindry D, Nielsen-Saines K, Chaves MC, Santos B, et al. Barriers and facilitators for men to attend prenatal care and obtain HIV voluntary counseling and testing in Brazil. PLoS ONE [Internet]. 2017 [acesso em 28 mar 2019]; 12(4). Disponível em: https://doi.org/10.1371/journal. pone.0175505.

20. Silva ARC, Falleiros AC, Vargas CRR, Turato ER, Lopes MHB de M. Discrimination Against Women Integrative Literature Review. Revista da ABPN [Internet]. 2015 [acesso em 28 jan 2019]; 7(15). Disponível em: http://abpnrevista.org.br/revista/index.php/revistaabpn1/article/view/119. 


\section{COMO REFERENCIAR ESTE ARTIGO:}

Brito JGE de, Santos JM de J, Barreiro M do SC, Dantas D da S, Leite AM, Mendes RB. Participação do companheiro da gestante nas consultas de pré-natal: prevalência e fatores associados. Cogitare enferm. [Internet]. 2021 [acesso em "colocar data de acesso, dia, mês abreviado e ano"]; 26. Disponível em: http://dx.doi.org/10.5380/ ce.v26i0.75169.

Recebido em: $13 / 07 / 2020$

Aprovado em: 02/02/2021

Editora associada: Tatiane Herreira Trigueiro

\section{Autor Correspondente:}

José Marcos de Jesus Santos

Universidade de São Paulo - Ribeirão Preto, SP, Brasil

E-mail: jsmarcos@usp.br

\section{Contribuição dos autores:}

Contribuições substanciais para a concepção ou desenho do estudo; ou a aquisição, análise ou interpretação de dados do estudo - JGEB, JMJS, RBM

Elaboração e revisão crítica do conteúdo intelectual do estudo - JGEB, JMJS, MSCB, DSD, AML, RBM

Aprovação da versão final do estudo a ser publicado - JGEB, JMJS, MSCB, DSD, AML, RBM

Responsável por todos os aspectos do estudo, assegurando as questões de precisão ou integridade de qualquer parte do estudo - JGEB, JMJS, RBM

Copyright $\odot 2021$ Este é um artigo em acesso aberto distribuído nos termos da Licença Creative Commons Atribuição, que permite o uso irrestrito, a distribuição e reprodução em qualquer meio desde que o artigo original seja devidamente citado. 